\title{
DESEMPENHO DE DIFERENTES ESPÉCIES DE Trichogramma (HYMENOPTERA: TRICHOGRAMMATIDAE) EM OVOS DE Spodoptera eridania (CRAMER) (LEPIDOPTERA: NOCTUIDAE)
}

\author{
CARVALHO, José Romário ${ }^{1}$ \\ PRATISSOLI, Dirceu $^{2}$ \\ ZAGO, Hugo Bolsoni ${ }^{3}$ \\ SOUZA, Lauana Pellanda de ${ }^{4}$ \\ OLIVEIRA, Carlos Magno Ramos ${ }^{5}$ \\ SANTOS, Vinícius Pereira dos ${ }^{4}$ \\ SALOMÃO, Kharen Priscilla de Oliveira Silva ${ }^{4}$
}

ISSUE DOI: $10.3738 / 1982.2278 .769$

\begin{abstract}
RESUMO: O objetivo deste trabalho foi avaliar o desempenho dos parasitoides Trichogramma pretiosum Riley, Trichogramma pratissolii Querino e Zucchi, Trichogramma exiguum Pinto e Platner, Trichogramma atopovirilia Oatman e Platner e Trichogramma galloi Zucchi (Hymenoptera: Trichogrammatidae) em ovos de Spodoptera eridania (Cramer) (Lepidoptera: Noctuidae). Foram utilizadas duas populações de T. pretiosum (comercial e de criação de laboratório) e uma população das demais espécies foram utilizadas no experimento. Cartelas azul celeste, contendo 20 ovos (idade $<12 \mathrm{~h}$ ) de $S$. eridania foram expostas ao parasitismo por 24 horas. Foram avaliados os seguintes parâmetros biológicos: número de ovos parasitados, percentual de emergência, número de indivíduos por ovo, razão sexual, duração do ciclo ovo-adulto e longevidade dos descendentes. O parasitismo diferiu entre as espécies de Trichogramma, variando entre 3,85 (19,25\%) e 9,90 (49,50\%) ovos parasitados por fêmea. A percentagem de emergência foi superior a $75 \%$ em todas as espécies/populações. A razão sexual foi maior para $T$. pretiosum população Tbug $(0,99)$, enquanto que o menor valor foi observado para T. atopovirilia e T. exiguum com 0,77 e 0,79 , respectivamente. A duração do ciclo ovo-adulto foi menor para as espécies T. pretiosum população Tbug, T. pratissolii e T. atopovirilia (9,00 dias). Os descendentes da população Tbug foram os que apresentaram maior longevidade (2,85 dias). Diante dos resultados de parasitismo, as espécies T. pretiosum e T. pratissolii demonstraram melhores índices, sendo desta forma, promissoras para a utilização em programa de controle biológico de S. eridania.
\end{abstract}

Palavras-chave: Inseto-praga. Controle biológico. Parasitoide de ovos.

\section{PERFORMANCE OF DIFFERENT SPECIES OF Trichogramma (HYMENOPTERA: TRICHOGRAMMATIDAE) IN EGGS Spodoptera eridania (CRAMER) (LEPIDOPTERA: NOCTUIDAE)}

\begin{abstract}
SUMMARY: The objective of this study was to evaluate the performance of the parasitoid Trichogramma pretiosum Riley, Trichogramma pratissolii Querino and Zucchi, Trichogramma exiguum Pinto and Platner, Trichogramma atopovirilia Oatman and Platner and Trichogramma galloi Zucchi (Hymenoptera: Trichogrammatidae) on eggs of Spodoptera eridania (Cramer) (Lepidoptera : Noctuidae). We used two populations of $T$. pretiosum (commercial and laboratory rearing) and a population of other species were used in the experiment. Cards azure, containing 20 eggs (age $<12$ h) of S. eridania were exposed for 24 hours of parasitism. We evaluated the following biological parameters: number of eggs parasitized, percentage of emergence, number of individuals per egg, sex ratio, duration of egg-adult and longevity of the offspring. The parasitism differed among Trichogramma species, ranging from $3.85(19.25 \%)$ and $9.90(49.50 \%)$ parasitized eggs per female. The emergence percentage was higher than $75 \%$. The sex ratio was higher for $T$. pretiosum population Tbug $(0.99)$, while the lower observed value for $T$. atopovirilia and T. exiguum, 0.77 and 0.79 , respectively. The time from egg to adult was lower for the species

\footnotetext{
1 Universidade Federal do Espírito Santo (UFES). Departamento de Produção Vegetal, Núcleo de Manejo Fitossanitário de Pragas e Doenças (NUDEMAFI), Mestre em Produção Vegetal - Fitossanidade.

${ }^{2}$ UFES. Departamento de Produção Vegetal, Núcleo de Manejo Fitossanitário de Pragas e Doenças (NUDEMAFI), Dr em Entomologia, Professor associado III.

${ }^{3}$ UFES. Departamento de Produção Vegetal, Nucleo de Manejo Fitossanitário de Pragas e Doenças (NUDEMAFI), Dr em Entomologia, Professor adjunto I

${ }^{4}$ UFES. Mestranda em Produção Vegetal.

${ }^{5}$ UFES. Doutorando em Produção Vegetal.
} 
T. pretiosum population Tbug, T. pratissolii and T. atopovirilia (9.00 days, respectively). The descendants of the population Tbug were those with greater longevity (2.85 days). Considering the results of parasitism, the species $T$. pretiosum and $T$. pratissolii demonstrated better rates, and thus promising for use in biological control program for $S$. eridania.

Keywords: Insect pest. Biological control. Egg parasitoid.

\section{INTRODUÇÃO}

Spodoptera eridania (Cramer) (Lepidoptera: Noctuidae) é uma praga polífaga que provoca desfolha em culturas economicamente importantes para o Brasil, como algodão e soja na região Centrooeste (SANTOS et al., 2005), tomate no Espírito Santo (PRATISSOLI, 2009) entre outras como milho, sorgo, hortaliças, frutíferas (KING; SANDERS, 1984), feijão (SAVOIE, 1988), maçã (NORA; REISFILHO; STUKER, 1989), folhas, folhagens, batatinha (GALLO et al., 2002), brássicas (MICHEREFFFILHO et al., 2008) e crotalária (DIAS et al., 2009).

Nos ínstares iniciais ( $1^{\circ}$ e $2^{\circ}$ ínstares) as lagartas de $S$. eridania apresentam hábito gregário enquanto que a partir do $3^{\circ}$ ínstar são solitárias. As características principais dos danos causados por este lepidóptero são o esqueletamento ou a desfolha (CAPINERA, 2001). O consumo foliar de S. eridania, no $5^{\circ}$ e $6^{\circ}$ ínstares chega a $104,3 \mathrm{~cm}^{2}$ quando criados em folhas de couve (LOPES; DELLA LUCIA; PICANÇO, 1997). Em algodoeiro, seu consumo, quando no $6^{\circ}$ instar, pode chegar a $80 \mathrm{~cm}^{2}$ (SANTOS et al., 2010), enquanto que em soja este valor se oscila entre 86,9 e $107,2 \mathrm{~cm}^{2}$ dependendo da cultivar (BUENO et al., 2011).

O controle dessa praga é realizado por meio de controle químico (MICHEREFF-FILHO et al., 2008). No entanto, muitos desses produtos não são registrados para este lepidóptero em culturas como o tomateiro (PRATISSOLI, 2009), portanto, o uso de agentes biológicos, como parasitoides, tem demonstrado potencialidade para o controle desse inseto-praga (SANTOS et al., 2010; GOULART et al., 2011), uma vez que os parasitoides são espécies-chave para a regulação de populações de insetos (QUERINO; ZUCCHI; PINTO, 2010).

Diversas espécies de parasitoides são usadas para o manejo de pragas em campo, e as espécies pertencentes ao gênero Trichogramma Westwood (Hymenoptera: Trichogrammatidae) merecem destaques, pois parasitoides desse gênero já foram relatadas parasitando mais de 200 espécies de insetospragas de várias culturas, principalmente da ordem Lepidoptera (DELPUECH; DUPONT; ALLEMAND, 2010; DAVIES; PUFKE; ZALUCKI, 2011). Ao longo da vida de T. pretiosum uma fêmea pode parasitar em média 13,8 ovos de Bonagota salubricola (Meyrick) (Lepidoptera: Tortricidae) a $25^{\circ} \mathrm{C}$ (PASTORI et al., 2007). Pratissoli et al. (2004) reportaram para esta mesma temperatura uma média de 21,6 ovos de Plutella xylostella (Linneau) (Lepitoptera: Plutellidae) parasitados por fêmea de T. pretiosum. Em Sitotroga cerealella (Olivier) (Lepidoptera: Gelechiidae), T. pretiosum e T. acacioi Brun, Moraes e Soares parasitaram em média 37,6 e 44,7 ovos por fêmea, respectivamente, a $25^{\circ} \mathrm{C}$ (PRATISSOLI et al., 2008).

No entanto, o sucesso ou fracasso da utilização de Trichogramma em programas de controle biológico é fundamentado pelo conhecimento de parâmetros biológicos desse parasitoide quando associado ao hospedeiro alvo, tais como: parasitismo, viabilidade, duração do ciclo, razão sexual e longevidade (SCHOLLER; HASSAN, 2001; BUENO; PARRA; BUENO, 2009). O conhecimento dessas características biológicas permite, entre outros aspectos, selecionar a espécie e/ou população do parasitoide que apresente maiores índices biológicos para controle de determinada praga (MILANEZ et al., 2009).

Diante do exposto, o objetivo desse trabalho foi avaliar o desempenho de diferentes espécies de 
Trichogramma com a finalidade de selecionar a de maior potencial para o manejo fitossanitário de $S$. eridania.

\section{MATERIAL E MÉTODOS}

Os ensaios foram realizados no setor de Entomologia do Núcleo de Desenvolvimento Científico e Tecnológico em Manejo Fitossanitário de Pragas e Doenças (NUDEMAFI), do Centro de Ciências Agrárias da Universidade Federal do Espírito Santo (CCA-UFES), Alegre - ES. Os ensaios e as criações dos insetos parasitoides do gênero Trichogramma spp. e S. eridania foram realizados em câmara climatizada, reguladas à temperatura de $25 \pm 1{ }^{\circ} \mathrm{C}$, umidade relativa de $70 \pm 10 \%$ e fotofase de 14 horas.

Coleta e Criação de $S$. eridania. Lagartas foram coletadas em lavouras comerciais de tomate e repolho localizadas no Distrito de São Paulinho, Domingos Martins, ES (Altitude: $620 \mathrm{~m}$; Latitude: 20²1'28" Sul; Longitude: 40³9'33" Oeste). As lagartas coletas foram alimentadas em laboratório com folhas de couve Brassica oleraceae cultivar Manteiga até atingirem a fase adulta. Após a emergência, os adultos foram mantidos em gaiolas de PVC (20,0 cm de diâmetro x 25,0 cm de altura) revestidos internamente com folha de papel branco para oviposição, sendo a extremidade superior fechada com tecido do tipo "voil" e a inferior fechada com uma placa quadrada de isopor $(25,0 \mathrm{~cm}$ de lado x $3,0 \mathrm{~cm}$ de espessura). Para as mariposas foram oferecidos diariamente um substrato alimentar a base de uma solução de mel a $10 \%(\mathrm{~m} / \mathrm{v})$, por meio de algodão embebido colocado em frasco de vidro $(5,0 \mathrm{~mL})$. As posturas foram removidas recortando o papel onde estavam as massas de ovos, sendo estas acondicionadas em Gerbox (6,0 cm de diâmetro x 1,5 cm de altura). As lagartas recém-eclodidas, foram inoculadas (2 lagartas por tubo) em tubos de vidro (2,5 cm de diâmetro x 10,0 cm de altura), contendo dieta artificial (GREENE et al., 1976), até a fase de pupa. As pupas foram coletadas dos tubos e transferidas para caixas plásticas (10,0 cm de comprimento x $10,0 \mathrm{~cm}$ de largura x $5,0 \mathrm{~cm}$ de altura) até a emergência dos adultos.

Obtenção e manutenção de Trichogramma spp. As espécies de parasitoides foram obtidas na coleção do NUDEMAFI/CCA-UFES, onde eram mantidas em ovos do hospedeiro Anagasta kuehniella (Zeller) (Lepidoptera: Pyralidae) (PRATISSOLI et al., 2010). Ovos desse hospedeiro foram colados com goma arábica diluída a 10,0\% (m/v) em retângulos de cartolina azul celeste (cartelas) $(4,0 \mathrm{~cm}$ de comprimento x 2,0 cm de largura) e oferecidos aos parasitoides em recipientes de vidro $(8,5 \mathrm{~cm}$ de comprimento $\mathrm{x} 2,4 \mathrm{~cm}$ de diâmetro), onde foi permitido o parasitismo por 24 horas. As espécies/populações de Trichogramma utilizadas no experimento estão descritas na Tabela 1.

Tabela 1 - Espécies de Trichogramma obtidas no trichogrammatério do NUDEMAFI/CCA-UFES com os respectivos locais de coleta/fornecedor.

(Continua)

\begin{tabular}{|c|c|c|c|}
\hline Espécies & Abreviação & Coletor & Local de coleta/Fornecedor \\
\hline $\begin{array}{l}\text { Trichogramma } \\
\text { pretiosum Riley }\end{array}$ & Tbug & - & BUG Agentes Biológicos, Piracicaba, SP \\
\hline $\begin{array}{l}\text { Trichogramma } \\
\text { pretiosum Riley }\end{array}$ & Tp14 & $\begin{array}{l}\text { P. L. } \\
\text { Pastori }\end{array}$ & $\begin{array}{c}\text { Sítio Nossa Senhora Aparecida, Pedra Preta, } \\
\text { MT }\end{array}$ \\
\hline $\begin{array}{l}\text { Trichogramma } \\
\text { galloi Zucchi }\end{array}$ & $\mathrm{Tg}$ & - & BUG Agentes Biológicos, Piracicaba, SP \\
\hline $\begin{array}{c}\text { Trichogramma } \\
\text { exiguum Pinto e } \\
\text { Platner }\end{array}$ & $\mathrm{Te}$ & $\begin{array}{l}\text { F. F. } \\
\text { Pereira }\end{array}$ & Distrito de Piaçu, Muniz Freire, ES \\
\hline
\end{tabular}


Tabela 1 - Espécies de Trichogramma obtidas no trichogrammatério do NUDEMAFI/CCA-UFES com os respectivos locais de coleta/fornecedor.

(Conclusão)

\begin{tabular}{|c|c|c|c|}
\hline $\begin{array}{c}\text { Trichogramma } \\
\text { atopovirilia } \\
\text { Oatman e } \\
\text { Platner }\end{array}$ & Tat & I. Cruz & $\begin{array}{c}\text { Centro Nacional de Pesquisa de Milho e } \\
\text { Sorgo, Sete Lagoas, MG }\end{array}$ \\
\hline $\begin{array}{c}\text { Trichogramma } \\
\text { pratissolii } \\
\text { Querino e } \\
\text { Zucchi }\end{array}$ & Tpr & T. G. Kloss & $\begin{array}{c}\text { Estação Biológica de Santa Lúcia, Santa } \\
\text { Teresa, ES. }\end{array}$ \\
\hline
\end{tabular}

Desempenho de Trichogramma spp. em ovos de $S$. eridania. Utilizou-se a metodologia adotada por de Bueno et al. (2008). Para cada espécie/população de Trichogramma foram utilizadas 20 fêmeas recém-emergidas (idade $<6$ horas) individualizadas em tubos de vidro $(4,0 \mathrm{~cm}$ de comprimento x $1,0 \mathrm{~cm}$ largura), sendo fechados com filme plástico PVC. Como alimento foi oferecido gotículas de mel distribuídas na parede interna dos tubos. Cada fêmea constituiu uma repetição e recebeu 20 ovos de $S$. eridania (idade < 12 horas), colados com goma arábica diluída a 10,0\% (m/v) em um retângulo de cartolina (3,5 cm de comprimento x 0,5 cm de diâmetro) de cor azul-celeste. As cartelas foram expostas ao parasitismo por 24 horas. Ao término desse período, as cartelas foram retiradas e acondicionadas em tubos de vidro $(8,5 \mathrm{~cm}$ de altura $\mathrm{x} 2,4 \mathrm{~cm}$ de diâmetro) e mantidas em câmaras climatizadas, até a emergência dos descendentes.

Foram avaliados os seguintes parâmetros: numero de ovos parasitados por fêmea, porcentagem de parasitismo e de emergência, número de indivíduos por ovo, razão sexual, duração do ciclo ovo-adulto e longevidade dos descendentes. Para a avaliação da longevidade dos descendentes retirou-se grupos de cinco indivíduos recém-emergidos em tubos de vidros fechados com filme plástico PVC, sem alimento, que permaneceram até a confirmação de suas mortes nas mesmas condições climáticas. Cada grupo de cinco indivíduos compôs uma repetição.

Análise dos dados. $\mathrm{O}$ delineamento utilizado foi o inteiramente casualizado com seis tratamentos (espécies/população de parasitoides) e 20 repetições. Os dados foram submetidos à análise de variância $(\mathrm{p} \leq 0,05)$ e as médias comparados pelo teste de Tukey $(\mathrm{p} \leq 0,05)$.

\section{RESULTADOS E DISCUSSÃO}

O parasitismo diferiu estatisticamente entre as espécies/populações ( $\mathrm{F}=19,722$; $\mathrm{GL}=6,114 ; \mathrm{p}<$ 0,001), variando entre 3,85 (19,25\%) e 9,90 (49,50\%) ovos parasitados por fêmea (Tabela 2). O maior valor observado de parasitismo $(9,90)$ foi encontrado para $T$. pretiosum (Tbug) sendo superior ao reportado por Goulart et al. (2011) para T. pretiosum em ovos de S. eridania em 24 horas de parasitismo (35\% de parasitismo). Isso demonstra que o hospedeiro, S. eridania, apresentou condições físico-químicas adequadas para o desenvolvimento das espécies/populações de Trichogramma estudadas. Além disso, as espécies $T$. pretiosum, populações Tbug e Tp14, e T. pratissolii foram as que apresentaram as maiores taxas de parasitismo, característica desejável para a utilização desse parasitoide em programas de manejo integrado de pragas. Além disso, apesar da população de T. pretiosum Tp14 ser uma população criada por várias gerações em laboratório, essa apresentou parasitismo estatisticamente semelhante ao observado para a população Tbug, que é produzido comercialmente. Isso demonstra que em condições laboratoriais pode se obter um agente biológico com características biológicas adequadas ao padrão de qualidade. 
Tabela 2 - Número de ovos parasitados por fêmea, percentual de emergência, número de descendentes emergidos por ovo e razão sexual de Trichogramma spp. em ovos de S. eridania. (Temperatura $25 \pm 1{ }^{\circ} \mathrm{C}$; umidade relativa de $70 \pm 10 \%$ e fotofase 14 horas)

\begin{tabular}{ccccc}
\hline Espécie/população & $\begin{array}{c}\text { Parasitismo } \\
(\mathbf{N}) *\end{array}$ & Parasitismo $(\%)$ & $\begin{array}{c}\text { Emergência } \\
(\%) * *\end{array}$ & $\begin{array}{c}\text { Indivíduos/ovo } \\
(\mathbf{N}) * *\end{array}$ \\
\hline Tbug & $9,90 \mathrm{a}$ & 49,50 & 76,82 & 1,24 \\
Tpr & $9,20 \mathrm{ab}$ & 46,00 & 80,13 & 1,15 \\
$\mathrm{Tg}$ & $7,35 \mathrm{~b}$ & 36,75 & 84,75 & 1,32 \\
Tp14 & $8,25 \mathrm{ab}$ & 41,25 & 79,48 & 1,21 \\
Te & $5,05 \mathrm{c}$ & 25,25 & 85,27 & 1,15 \\
Tat & $3,85 \mathrm{c}$ & 19,25 & 81,36 & 1,02 \\
\hline F & 19,722 & --- & 0,4906 & 1,4631 \\
p & $<0,001$ & --- & $>0,050$ & 0,2074 \\
GLR & 114 & --- & 114 & 114 \\
CV $(\%)$ & 32,93 & --- & 25,43 & 31,9 \\
\hline
\end{tabular}

*Médias seguidas de mesma letra na coluna não diferem entre si pelo teste de Tukey $(\mathrm{p} \leq 0,05)$;

**Não significativo.

Quanto ao porcentual de emergência e ao número de indivíduos por ovo, esses parâmetros não sofreram variação significativa entre as espécies e/ou populações de Trichogramma $(\mathrm{F}=0,4906$; GL = 6,114; $\mathrm{p}=0,050$ para percentagem de emergência e; $\mathrm{F}=1,4631 ; \mathrm{GL}=6,114 ; \mathrm{p}=0,2074$ para número de indivíduos por ovo) (Tabela 2). Quando criados em S. eridania as diferentes espécies e/ou populações de Trichogramma, apresentaram porcentual médio de emergência de $81,30 \%$ e número de indivíduos por ovo superior a 1,0 indivíduos/ovo.

Os resultados de emergência estão dentro da faixa observada por Hassan e Zhang (2001) para Trichogramma brassicae Bezd produzido comercialmente na Alemanha para o controle de Ostrinia nubilalis (Hübner) (Lepidoptera: Pyralidae) (72,3 a 90,6\% de emergência). Esse valor se encontra próximo de $85 \%$, valor considerado dentro do padrão de qualidade na produção desses inimigos naturais (ALMEIDA; SILVA; MEDEIROS, 1998). Contudo, o valor encontrado no presente estudo é inferior ao relatado por Andrade et al. (2009), que verificaram taxas de emergência para T. pretiosum, T. atopovirilia e T. exiguum superiores a 91\% em ovos de Heliothis virescens (Fabricius) (Lepidoptera: Noctuidae).

Para o número de indivíduos por ovo, o valor médio observado no presente estudo $(1,18$ indivíduos/ovo) é inferior ao relatado por Andrade et al. (2009), que obtiveram valores superiores a 1,7 indivíduos/ovo para $T$. pretiosum, T. atopovirilia e T. exiguum criados em ovos de H. virescens. No entanto, foi próximo aos valores reportados por Meira et al. (2009) (1,0 indivíduo/ovo) para T. pretiosum, T. atopovirilia, T. exiguum e T. acacioi em ovos de P. xylostella. Isso possivelmente deve esta diretamente sendo afetado pelo fato dos ovos de S. eridania $[0,45 \mathrm{~mm}$ de diâmetro x $0,35 \mathrm{~mm}$ de altura (CAPINERA, 2001)] serem menores que os ovos de $H$. virescens $[0,51-0,60 \mathrm{~mm}$ de largura $\mathrm{x}$ 0,50-0,61 mm de comprimento (CAPINERA, 2001)]. Com relação a P. xylostella, seus ovos [0,26 mm de largura x 0,44 mm de comprimento (CAPINERA, 2001)] são menores aos de S. eridania. Desta forma, tais variações encontradas podem estar relacionadas com as condições físico-químicas do ovo hospedeiro, pois o parasitoide ao analisar o ovo hospedeiro, pode verificar que ele é de baixa qualidade, ou seja, que dispõem de pouco alimento para as proles completarem o seu desenvolvimento, podendo assim reduzir o número de progênies colocados por ovo (VINSON, 2010).

A razão sexual diferiu significativamente entre os parasitoides $T$. pretiosum população Tbug e as espécies T. exiguum e T. atopovirilia $(\mathrm{F}=4,0432 ; \mathrm{GL}=6,114 ; \mathrm{p}=0,002)$ (Tabela 3). Os parasitoides $T$. 
pretiosum população Tp14 e T. galloi apresentaram valores intermediários (0,92 e 0,90 respectivamente). O elevado valor de fêmeas para $T$. pretiosum população Tbug $(0,99)$ pode estar associado, possivelmente, à melhor adequação dessa espécie para com o hospedeiro $S$. eridania. Em função disso, verificou-se semelhança para este parâmetro para a população Tp14, enquanto que para T. pratissolii, T. exiguum e T. atopovirilia necessitaram de machos, em hipótese, para que ocorra variabilidade genética por meio de cruzamento para melhor adaptação ao hospedeiro (BOIVIN, 2010; RUSSEL; STOUTHAMER, 2010).

Tabela 3 - Razão sexual, duração do ciclo ovo-adulto e longevidade dos descendentes de Trichogramma spp. criados em ovos de $S$. eridania. (Temperatura $25 \pm 1{ }^{\circ} \mathrm{C}$; umidade relativa de $70 \pm 10 \%$ e fotofase 14 horas)

\begin{tabular}{cccc}
\hline Espécie/população & Razão sexual* $^{*}$ & Ciclo (dias)* & $\begin{array}{c}\text { Longevidade dos } \\
\text { descendentes (dias)* }\end{array}$ \\
\hline Tbug & $0,99 \mathrm{a}$ & $9,00 \mathrm{c}$ & $2,85 \mathrm{a}$ \\
$\mathrm{Tpr}$ & $0,79 \mathrm{~b}$ & $9,00 \mathrm{c}$ & $1,15 \mathrm{c}$ \\
$\mathrm{Tg}$ & $0,90 \mathrm{ab}$ & $9,80 \mathrm{a}$ & $1,85 \mathrm{bc}$ \\
$\mathrm{Tp} 14$ & $0,92 \mathrm{ab}$ & $10,00 \mathrm{a}$ & $2,00 \mathrm{~b}$ \\
$\mathrm{Te}$ & $0,79 \mathrm{~b}$ & $9,50 \mathrm{~b}$ & $1,75 \mathrm{bc}$ \\
$\mathrm{Tat}$ & $0,77 \mathrm{~b}$ & $9,00 \mathrm{c}$ & $1,35 \mathrm{bc}$ \\
\hline $\mathrm{F}$ & 4,0432 & 56,0732 & 8,9666 \\
$\mathrm{p}$ & 0,002 & $<0,001$ & $<0,001$ \\
$\mathrm{GLR}$ & 114 & 114 & 114 \\
$\mathrm{CV}(\%)$ & 23,12 & 2,86 & 48,67 \\
\hline
\end{tabular}

*Médias seguidas de mesma letra nas colunas não diferem estatisticamente pelo teste de Tukey $(\mathrm{p} \leq 0,05)$.

A produção de uma maior quantidade de fêmeas é muito importante em se tratando da utilização do Trichogramma em programas de controle biológico (HASSAN; ZHANG, 2001). Contudo, as condições adversas dos agroecossistemas promovem a necessidade de adaptabilidade desses parasitoides, o que ocorre naturalmente por meio dos cruzamentos entre machos e fêmeas. Além disso, a constituição nutricional do ovo hospedeiro pode influenciar a produção de machos e fêmeas (VINSON, 1997). Ao estudar linhagens de $T$. pretiosum criadas em ovos de Grapholita molesta (Busck) (Lepidoptera: Tortricidae), Rodrigues et al. (2011) reportaram uma grande variação na razão sexual $(0,31$ - 0,77), demonstrando que entre populações da mesma espécie pode haver diferença quanto à produção de machos e fêmeas.

A duração do ciclo ovo-adulto para T. pretiosum população Tbug, T. pratissolii e T. atopovirilia foram iguais $(9,00$ dias, respectivamente para cada parasitóide) $(\mathrm{F}=56,0732 ; \mathrm{GL}=6,114 ; \mathrm{p}<0,001)$, enquanto que $T$. pretiosum população Tp14 e $T$. galloi foram estatisticamente superiores ao dos demais (10,00 e 9,80 dias, respectivamente) (Tabela 3). Uma menor duração do ciclo ovo-adulto é uma característica desejável para programas de controle biológico com parasitoides, pois em um menor tempo pode-se obter novas populações aptas em campo.

O tempo requerido para o desenvolvimento ovo-adulto para as populações de Trichogramma estudadas foram semelhantes ao observado por Beserra, Dias e Parra (2003), para diferentes populações/ linhagens de T. pretiosum em ovos de Spodoptera frugiperda (Smith) (Lepidoptera: Noctuidae). Todavia, os resultados também foram inferiores aos relatados por Bueno, Parra e Bueno (2009), para T. pretiosum em ovos de Anticarsia gemmatalis (Hübner) e Chrysodeixis (Pseudoplusia) includens (Walker) (Lepidoptera: Noctuidae) (10,3 e 10,0 dias), exceto para o T. pretiosum população Tp14 que apresentou ciclo de desenvolvimento de 10 dias. Em função das pesquisas já realizadas em relação à duração do ciclo 
de desenvolvimento, pode-se dizer que a variação ocorrida neste parâmetro foi influenciada pela espécie e, ou população de Trichogramma.

A longevidade dos descendentes diferiu estatisticamente entre as espécies e, ou populações de Trichogramma estudadas ( $\mathrm{F}=8,9666 ; \mathrm{GL}=6,114 ; \mathrm{p}<0,001$ ) (Tabela 3), sendo a maior longevidade observada para os descendentes de Tbug (2,85 dias). Isso possivelmente deve ter ocorrido devido a uma melhor adaptabilidade de $T$. pretiosum população Tbug quanto ao hospedeiro $S$. eridania. A espécie $T$. pratissolii foi a que apresentou o menor valor de longevidade dos descendentes (1,15 dias). A longevidade é uma característica importante que deve ser levada em consideração nos programas de controle biológico, pois quanto maior for o tempo de vida, os parasitoides provavelmente serão mais eficientes no campo e capazes de parasitar mais ovos de insetos-praga (GOMES, 1997; RODRIGUES et al., 2011).

O estudo de diferentes espécies Trichogramma sobre um determinado lepidóptero-praga é de suma importância, pois proporciona a obtenção de informações fundamentais para a seleção da(s) melhor (es) espécie (s)/população (ões) para o manejo fitossanitário de pragas.

\section{CONCLUSÃO}

1) As populações Tbug e Tp14 de T. pretiosum e a espécie $T$. pratissolii foram as que apresentaram maiores taxas de parasitismo;

2) Os parâmetros porcentagem de emergência e número de indivíduos/ovo não foram influenciados pelo hospedeiro;

3) Independente da espécie/população de Trichogramma a proporção de fêmeas foi superior a de machos

4) A duração do ciclo ovo-adulto foi menor para as espécies $T$. pretiosum população Tbug e $T$. pratissolii, enquanto que os descendentes de T. pretiosum população Tbug apresentaram maior longevidade;

5) Diante do exposto as espécies $T$. pretiosum população Tbug e $T$. pratissolii, demonstraram melhores resultados, apontando-as como promissoras para a utilização em programas de manejo integrado de S. eridania.

\section{AGRADECIMENTOS}

Às instituições de fomento FINEP, CNPq e FAPES pelo financiamento.

\section{REFERÊNCIAS}

ALMEIDA, R. P.; SILVA, C. A. D.; MEDEIROS, M. B. (Ed.). Biotecnologia de produção massal e manejo de Trichogramma para o controle biológico de pragas. Campina Grande: Embrapa-CMPA, 1998

ANDRADE, G. S.et al. Parasitismo de ovos de Heliothis virescens por Trichogramma spp. pode ser afetado por cultivares de algodão. Acta Scientiarum Agronomy, v. 31, n. 4, p. 569-573, 2009.

BESERRA, E. B.; DIAS, C. T. S.; PARRA, J. R. P. Características biológicas de linhagens de Trichogramma pretiosum desenvolvidas em ovos de Spodoptera frugiperda. Acta Scientiarum, v. 25, p. 479-483, 2003.

BOIVIN, G. Reproduction and immature development of egg parasitoids. In: CÔNSOLI, F. L.; PARRA, J. R. P.; ZUCCHI, R. (Eds.) Egg Parasitoids in Agroecosystems with Emphasis on Trichogramma. Springer, London, p. 1-26, 2010. 
BUENO, R. C. O. F.et al. Lepidopteran larva consumption of soybean foliage: basis for developing multiple-species economic thresholds for pest management decisions. Pest Management Science, v. 67, p. 170-174, 2011.

BUENO, R. C. O. F.; PARRA, J. R. P.; BUENO, A. F. Biological characteristics and thermal requirements of a Brazilian strain of the parasitoid Trichogramma pretiosum reared on eggs of Pseudoplusia includens and Anticarsia gemmatalis. Biological Control, v. 51, p. 355-361, 2009.

CAPINERA, J. L. Handbook of vegetable pest. ACADEMIC PRESS, New York, 2001. 762 p.

DAVIES, A. P.; PUFKE, U. S.; ZALUCKI, M. P. Trichogramma (Hymenoptera: Trichogrammatidae) Ecology in a tropical Bt transgenic cotton cropping cystem: Sampling to improve seasonal pest impact estimates in the Ord River Irrigation Area, Australia. Journal Economic Entomological, v. 102, n. 3, p. 1018-1031, 2009.

DELPUECH, J. M.; DUPONT, C.; ALLEMAND, R. Decrease in fecundity induced by interspecific mating between two Trichogramma parasitoid species. Journal Economic Entomology, v. 103, n. 2, p. 308-313, 2010.

DIAS, N. S. et al. Primeiro registro de ocorrência de Spodoptera spp. (Lepidoptera: Noctuidae) atacando crotalária no estado de Alagoas, Brasil. Revista Caatinga, v. 22, n. 1, p. 01-03, 2009.

GALLO, D. et al. (eds.), Manual de Entomologa Agrícola, FEALQ, 2002, 920p.

GOMES, S. M. Comparação de três hospedeiros alternativos para a criação e produção massal de Trichogramma pretiosum Riley, 1879 e Trichogramma galloi Zucchi. 1997. 106 f. Dissertação (mestrado). Escola Superior de Agricultura "Luiz de Queiroz", Universidade de São Paulo. Piracicaba.

GOULART, M. M. P.et al. Host preference of the egg parasitoids Telenomus remus and Trichogramma pretiosum in laboratory. Revista Brasileira de Entomologia, v. 55, n. 1, p. 129-133, 2011.

GREENE, G. L.; LEPPLA, N. C.; DICKERSON, W. A. Velvetbean caterpillar: a rearing procedure and artificial medium. Journal Economic Entomology, n. 69, p. 487-497, 1976.

HASSAN, S. A.; ZHANG, W. Q. Variability in quality of Trichogramma brassicae (Hymenoptera: Trichogrammatidae) from commercial suppliers in Germany. Biological Control, v. 22, p. 115-121, 2001.

LOPES, C. M. D‘A.; DELLA LUCIA, T. M. C.; PICANÇO, M.C. Consumo foliar de lagartas de Spodoptera eridania (Cramer, 1782) parasitadas por Cyrtophloeba ESP.N. (Diptera: Tachinidae). Revista Brasileira de Biologia, 57, p.15 - 19, 1997.

MEIRA, A. L.et al. Seleção de espécies de Trichogramma sp. em ovos da traça-das-crucíferas, Plutella xylostella. Revista Caatinga, v. 24, n. 2, p. 1-8, 2011.

MICHEREFF-FILHO, M.et al. Effect of some biorational insecticides on Spodoptera eridania in organic cabbage. Pest Management Science, v. 64, n. 7, p. 761-767, 2008.

MILANEZ, A. M.et al. Avaliação de Trichogramma spp. para o controle de Trichoplusia ni. Pesquisa Agropecuária Brasileira, v. 44, n. 10, p. 1219-1224, 2009.

NORA, I.; REIS-FILHO, W.; STUKER, H. Danos de lagartas em frutos e folhas da macieira: Mudanças no agroecosistema ocasionam o surgimento de insetos indesejados nos pomares. Agropecuária Catarinense. v. 2, p. 54-55, 1989.

PRATISSOLI, D. Tomate: Spodoptera em tomate. Cultivar: Frutas e Hortaliças, v. 54, p. 6-7, 2009. 
PRATISSOLI, D.et al. Características biológicas de Trichogramma exiguum em ovos de Anagasta kuehniella e Sitotroga cerealella. Idésia, v. 28, n. 1, p. 39-42, 2010.

PRATISSOLI, D.et al. Efeito da ausência de hospedeiro e de alimento sobre aspectos biológicos de Trichogramma pretiosum Riley (Hymenoptera: Trichogrammatidae). Acta Scientiarum. Agronomy, v. 26, p. 281-286, 2004.

PRATISSOLI, D.; PARRA, J. R. P. Desenvolvimento e exigências térmicas de Trichogramma pretiosum Riley, criados em duas traças do tomateiro. Pesquisa Agropecuária Brasileira, n. 35, p. 1281-1288, 2000.

PRATISSOLI, D.et al. Parasitismo de Trichogramma pretiosum em ovos da traça-das-crucíferas sob diferentes temperaturas. Horticultura Brasileira, v.22, n.4, p.754-757, 2004.

PRATISSOLI, D. et al. Parasitism capacity of Trichogramma pretiosum and Trichogramma acacioi (Hym.: Trichogrammatidae) in eggs of Sitotroga cerealella (Lep.: Gelechiidae). Brazilian Archives of Biology and Technology, v. 51 n. 6, p. 1249-1254, 2008.

QUERINO, R. B.; ZUCCHI, R. A.; PINTO, J. D. Systematics of the trichogrammatidae (Hymenoptera: Chalcidoidea) with a focus on the genera attacking Lepidoptera. In: CÔNSOLI, F. L.; PARRA, J. R. P.; ZUCCHI, R. (Eds.) Egg Parasitoids in Agroecosystems with Emphasis on Trichogramma. Springer, London, p. 191-218, 2010.

RODRIGUES, M. L.et al. Selection of Trichogramma pretiosum lineages for control of Grapholita molesta in peach. Florida Entomologist, v. 94, n. 3, p. 398-403, 2011.

RUSSEL, J. E.; STOUTHAMER, R. Sex ratio madulators of egg parasioids. In: CÔNSOLI, F. L.; PARRA, J. R. P.; ZUCCHI, R. (Eds.) Egg Parasitoids in Agroecosystems with Emphasis on Trichogramma. Springer, London, p. 1-26, 2010.

SANTOS, K. B.et al. Caracterização dos danos de Spodoptera eridania (Cramer) e Spodoptera cosmioides (Walker) (Lepidoptera: Noctuidae) a estruturas de algodoeiro. Neotropical Entomology, v. 39 , n. 4, p. 626-631, 2010.

SANTOS, K. B.; NEVES, P. J.; MENEGUIM, A. M. Biologia de Spodoptera eridania (Cramer) (Lepidoptera: Noctuidae) em diferentes hospedeiros. Neotropical Entomology, v. 34, p. 903-910, 2005.

SAVOIE, K.L. Selective feeding by species of Spodoptera (Lepidoptera: Noctuidae) in a bean field with minimum tillage. Turrialba, v. 38, p. 67-70, 1988.

SCHOLLER, M.; HASSAN, S. A. Comparative biology and life tables of Trichogramma evanescens and T. cacoeciae with Ephestia elutella as host at four constant temperatures. Entomologia Experimentalis et Applicata, v. 98, p. 35-40, 2001.

VINSON, S. B. Nutritional ecology of insect egg parasitoids. In: CÔNSOLI, F. L.; PARRA, J. R. P.;

ZUCCHI, R. A. (Eds.). Egg parasitoids in agroecosystems with emphasis on Trichogramma. Springer, London, p. 25-56, 2010.

VINSON, S.B. Comportamento de seleção hospedeira de parasitoides de ovos, com ênfase na família Trichogrammatidae. In: PARRA, J. R. P., ZUCCHI, R. A. (Eds.), Trichogramma e o Controle Biológico Aplicado. FEALQ Piracicaba, p. 67-120, 1997.

VIRGALA, M. B. R.; BOTTO, E. N. Estudios biológicos de Trichogrammatoidea bactrae Nagaraja (Hymenoptera: Trichogrammatidae), parasitoide de huevos de Tuta absoluta Meyrick (Lepidoptera: Gelechiidae). Neotropical Entomology, v. 39, n. 4, p. 612-617, 2010. 
